The Open Pain Journal
Bentham OPen
CrossMark
DOntent list available at: $10.2174 / 1876386301710010001$

\title{
LETTER
}

\section{Antimicrobial Activity of Lidocaine, Bupivacaine, Mepivacaine and Ropivacaine on Staphylococcus epidermidis, Staphylococcus aureus and Bacillus subtilis}

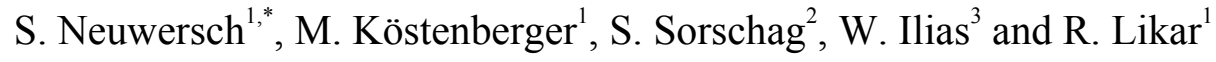 \\ ${ }^{I}$ Department of Anaesthesiology and Intensive Care, General Hospital Klagenfurt, Klagenfurt, Austria \\ ${ }^{2}$ Institute of Laboratory Diagnostics and Microbiology, General Hospital Klagenfurt, Klagenfurt, Austria \\ ${ }^{3}$ Vienna Private Clinic, Vienna, Austria
}

Received: August 24, 2016

Revised: January 04, 2017

Accepted: January 06, 2017

\begin{abstract}
:
Introduction:

Various studies have shown a possible antimicrobial activity of different local anaesthetics, which may affect the results of microbial assessment of biopsies. The purpose of this study was to test the antimicrobial activity of different commonly used anaesthetic agents on Staphylococcus epidermidis, Staphylococcus aureus and Bacillus subtitles to reproduce data and to compare the findings.
\end{abstract}

\section{Methods:}

Local anaesthetics tested were commercially available solutions of lidocaine (Xyloneural ${ }^{\circledR}$, Xylanaest pur. $\left.^{\circledR}\right)$, bupivacaine $\left(\right.$ Bucain $\left.^{\circledR}\right)$, mepivacaine $\left(\right.$ Mecain $\left.^{\circledR}\right)$ and ropivacaine $\left(\right.$ Naropin $^{\circledR}$, Ropinaest $\left.^{\circledR}\right) .2 \%, 1 \%, 0.5 \%, 0.25 \%$ and $0.1 \%(20,10,5,2.5,1 \mathrm{mg} / \mathrm{ml}) \mathrm{dilutions}$ of these local anaesthetics were prepared with sterile $0.9 \%$ saline. Bacteria used in this study were Staphylococcus epidermidis, Staphylococcus aureus and Bacillus subtilis. $10 \mu \mathrm{l}$ of different local anaesthetic dilution placed on thin wafers were added to Mueller Hinton Agar and cultured. After 24 hours, a zone of inhibition around the wafers was evaluated.

\section{Results:}

Local anaesthetics in different concentrations did not show any zone of inhibition on Staphylococcus epidermidis, Staphylococcus aureus or Bacillus subtilis.

\section{Conclusion:}

In summary, neither lidocaine, bupivacaine, mepivacaine nor ropivacaine showed an antibacterial effect on Staphylococcus epidermidis, Staphylococcus aureus and Bacillus subtilis.

\section{Implications:}

Due to these findings this local anaesthetics can be used in daily clinical routine to perform pain free diagnostic procedures in which culture specimens are to be obtained. Due to inconsistent results in prior studies, we recommend to use the lowest concentration possible of the local anaesthetic, also to avoid other possible side effects of local agents.

Keywords: Pain, Local anaestetic, Concentration, Antibacterial effect.

* Address correspondence to this author at the Department of Anaesthesiology and Intensive Care, Institution: General Hospital Klagenfurt, Klagenfurt, Austria; Tel: 0043/538/0; Email: stefan.neuwersch@kabeg.at 


\section{INTRODUCTION}

In clinical practice local anaesthetics are used daily to perform pain free diagnostic procedures. However, various studies have shown a possible antimicrobial activity of different local anaesthetics, which may affect results in microbial assessment of biopsies. The purpose of this study was to test the antimicrobial activity of different commonly used anaesthetic agents on Staphylococcus epidermidis, Staphylococcus aureus and Bacillus subtilis to reproduce data and to compare the findings.

\section{METHODS}

Local anaesthetics tested were commercially available solutions of lidocaine (Xyloneural ${ }^{\circledR}$, Xylanaest pur. ${ }^{\circledR}$ ), bupivacaine $\left(\right.$ Bucain $\left.^{\circledR}\right)$, mepivacaine $\left(\right.$ Mecain $\left.^{\circledR}\right)$ and ropivacaine $\left(\right.$ Naropin $^{\circledR}$, Ropinaest $\left.^{\circledR}\right) .2 \%, 1 \%, 0.5 \%, 0.25 \%$ and $0.1 \%(20,10,5,2.5,1 \mathrm{mg} / \mathrm{ml})$ dilutions of these local anaesthetics were prepared with sterile $0.9 \%$ saline. Bacteria used in this study were Staphylococcus epidermidis, Staphylococcus aureus and Bacillus subtilis. The number of bacteria was prepared to achieve $0.5 \mathrm{McFarland}$ standard and plated. $10 \mu \mathrm{l}$ of different local anaesthetic dilution placed on thin wafers were added to Mueller Hinton Agar and cultured at $35^{\circ} \mathrm{C}$. Wafers without local anaesthetics but $0.9 \%$ saline and wafers with ampicillin/sulbactam were used as controls. After 24 hours, a zone of inhibition around the wafers was evaluated, which is a wide ring around the wafer with no bacterial growth.

\section{RESULTS}

Local anaesthetics in different concentrations did not show any zone of inhibition on Staphylococcus epidermidis, Staphylococcus aureus or Bacillus subtilis, which means that these bacteria have grown although there was a local anaesthetic (Figs. 1 and 2). Due to these findings the tests were repeated with commercially available undiluted local anaesthetics but with lower densities of microorganisms, according to McFarland standard of 0.25, 0.10 and 0.05 and $100 \mu \mathrm{l}$ of different local anesthetic solution. After culturing for 24 hours at $35^{\circ} \mathrm{C}$ there again was no zone of inhibition on tested bacteria and no change in the surrounding bacterial concentration. In summary, 240 combinations of bacterial and local anaesthetics were tested (Table 1).

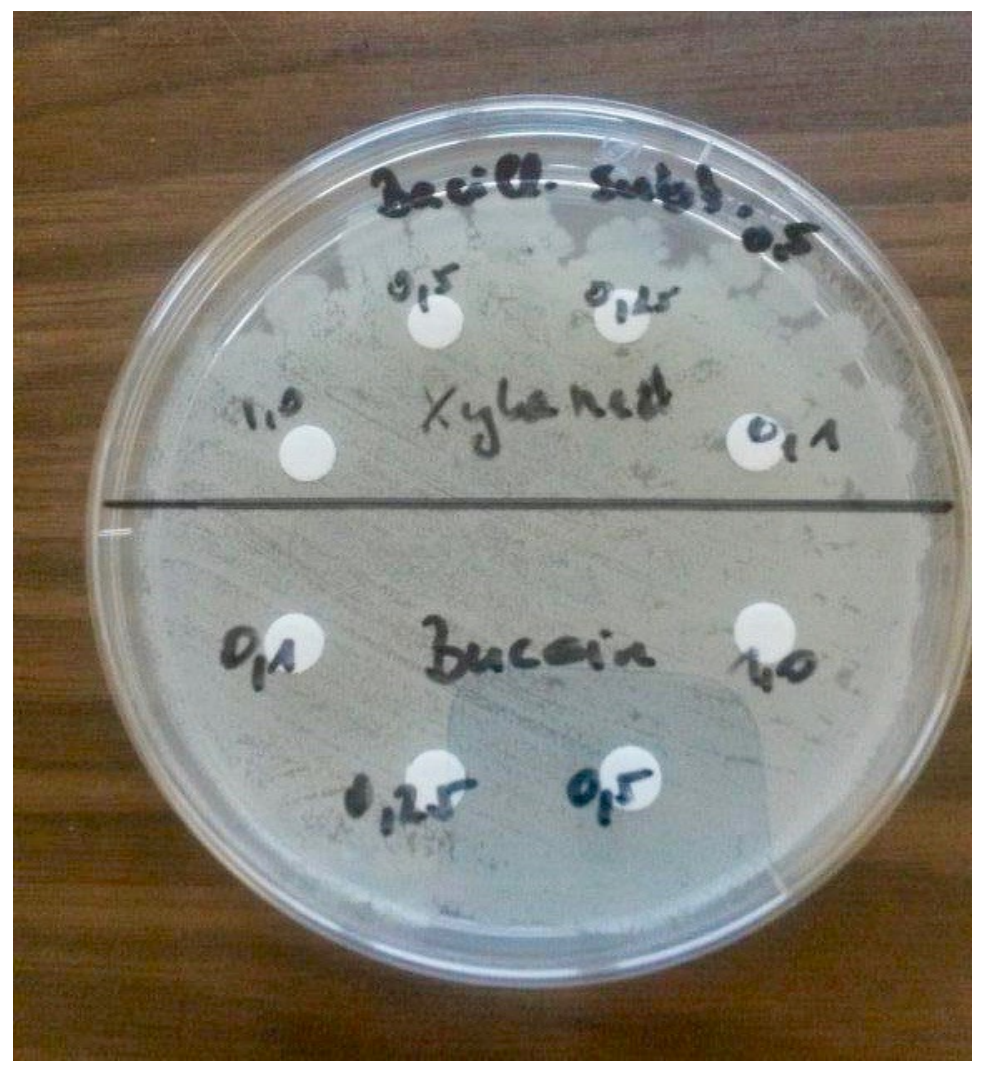

Fig. (1). Local anaesthetics in different concentrations did not show any zone of inhibition on Bacillus subtilis. 


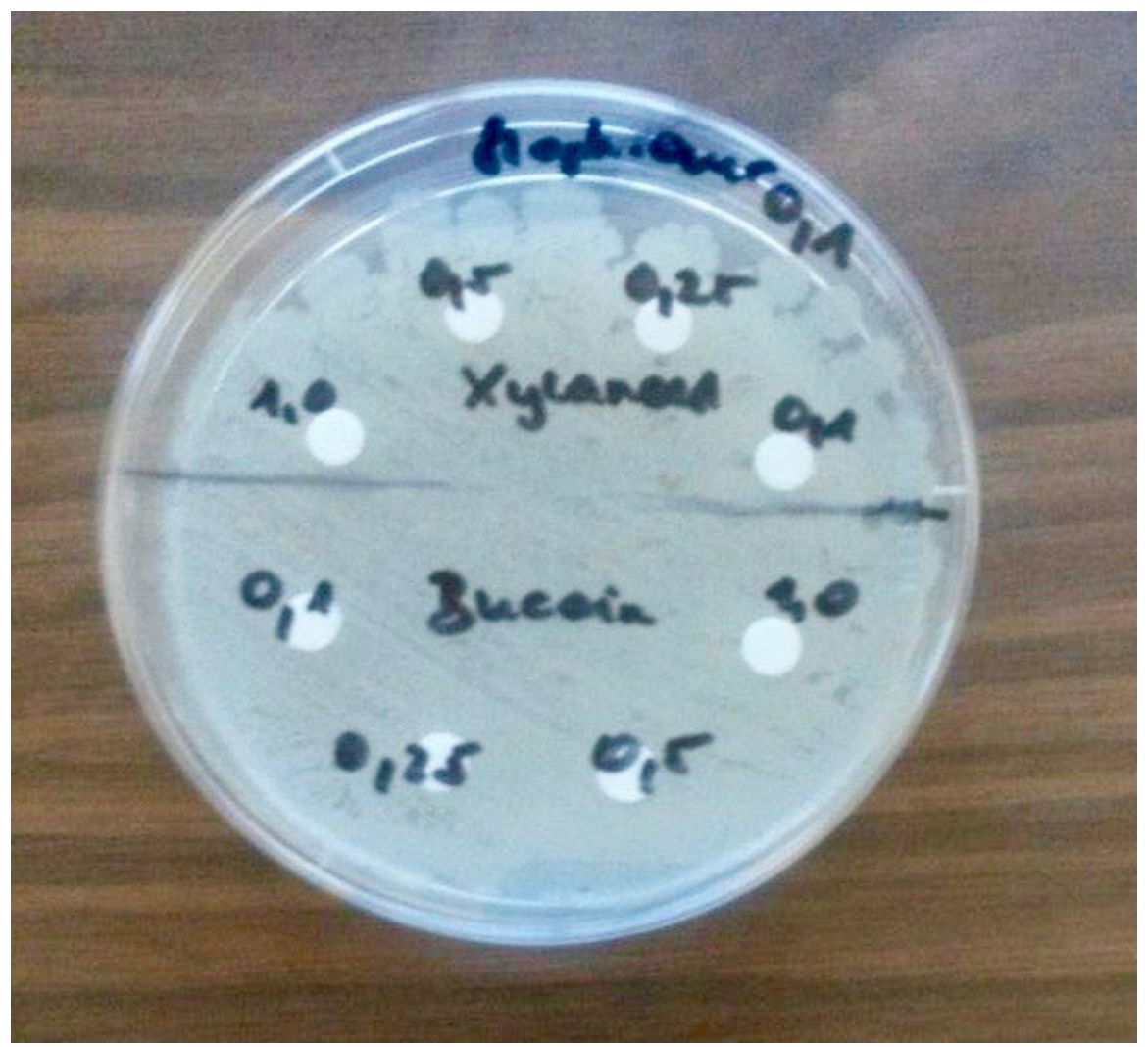

Fig. (2). Local anaesthetics in different concentrations did not show any zone of inhibition on Staphylococcus aureus.

Table 1. Local anaesthetics / zone of inhibition.

\begin{tabular}{|c|c|c|c|c|c|c|c|c|c|c|c|c|c|}
\hline \multirow{3}{*}{\begin{tabular}{|l|} 
Tested local \\
anaesthetics
\end{tabular}} & & \multicolumn{12}{|c|}{ Mc Farland standard } \\
\hline & & \multicolumn{4}{|c|}{$\begin{array}{c}\text { Staphylococcus } \\
\text { epidermidis }\end{array}$} & \multicolumn{4}{|c|}{$\begin{array}{c}\text { Staphylococcus } \\
\text { aureus }\end{array}$} & \multicolumn{4}{|c|}{$\begin{array}{l}\text { Bacillus } \\
\text { subtilis }\end{array}$} \\
\hline & conc. & $\mathbf{0 , 5 0}$ & 0,25 & 0,10 & 0,05 & $\mathbf{0 , 5 0}$ & 0,25 & $\mathbf{0 , 1 0}$ & 0,05 & $\mathbf{0 , 5 0}$ & 0,25 & 0,10 & 0,05 \\
\hline Xyloneural $^{\circledR}$ & 1,0 & $0 \mathrm{~mm}$ & $0 \mathrm{~mm}$ & $0 \mathrm{~mm}$ & $0 \mathrm{~mm}$ & $0 \mathrm{~mm}$ & $0 \mathrm{~mm}$ & $0 \mathrm{~mm}$ & $0 \mathrm{~mm}$ & $0 \mathrm{~mm}$ & $0 \mathrm{~mm}$ & $0 \mathrm{~mm}$ & $0 \mathrm{~mm}$ \\
\hline Xylanaest & 0,5 & $0 \mathrm{~mm}$ & $0 \mathrm{~mm}$ & $0 \mathrm{~mm}$ & $0 \mathrm{~mm}$ & $0 \mathrm{~mm}$ & $0 \mathrm{~mm}$ & $0 \mathrm{~mm}$ & $0 \mathrm{~mm}$ & $0 \mathrm{~mm}$ & $0 \mathrm{~mm}$ & $0 \mathrm{~mm}$ & $0 \mathrm{~mm}$ \\
\hline \multirow[t]{2}{*}{ pur ${ }^{(B)}$} & 0,25 & $0 \mathrm{~mm}$ & $0 \mathrm{~mm}$ & $0 \mathrm{~mm}$ & $0 \mathrm{~mm}$ & $0 \mathrm{~mm}$ & $0 \mathrm{~mm}$ & $0 \mathrm{~mm}$ & $0 \mathrm{~mm}$ & $0 \mathrm{~mm}$ & $0 \mathrm{~mm}$ & $0 \mathrm{~mm}$ & $0 \mathrm{~mm}$ \\
\hline & 0,1 & $0 \mathrm{~mm}$ & $0 \mathrm{~mm}$ & $0 \mathrm{~mm}$ & $0 \mathrm{~mm}$ & $0 \mathrm{~mm}$ & $0 \mathrm{~mm}$ & $0 \mathrm{~mm}$ & $0 \mathrm{~mm}$ & $0 \mathrm{~mm}$ & $0 \mathrm{~mm}$ & $0 \mathrm{~mm}$ & $0 \mathrm{~mm}$ \\
\hline \multirow[t]{4}{*}{ Bucain $^{\circledR}$} & 1,0 & $0 \mathrm{~mm}$ & $0 \mathrm{~mm}$ & $0 \mathrm{~mm}$ & $0 \mathrm{~mm}$ & $0 \mathrm{~mm}$ & $0 \mathrm{~mm}$ & $0 \mathrm{~mm}$ & $0 \mathrm{~mm}$ & $0 \mathrm{~mm}$ & $0 \mathrm{~mm}$ & $0 \mathrm{~mm}$ & $0 \mathrm{~mm}$ \\
\hline & 0,5 & $0 \mathrm{~mm}$ & $0 \mathrm{~mm}$ & $0 \mathrm{~mm}$ & $0 \mathrm{~mm}$ & $0 \mathrm{~mm}$ & $0 \mathrm{~mm}$ & $0 \mathrm{~mm}$ & $0 \mathrm{~mm}$ & $0 \mathrm{~mm}$ & $0 \mathrm{~mm}$ & $0 \mathrm{~mm}$ & $0 \mathrm{~mm}$ \\
\hline & 0,25 & $0 \mathrm{~mm}$ & $0 \mathrm{~mm}$ & $0 \mathrm{~mm}$ & $0 \mathrm{~mm}$ & $0 \mathrm{~mm}$ & $0 \mathrm{~mm}$ & $0 \mathrm{~mm}$ & $0 \mathrm{~mm}$ & $0 \mathrm{~mm}$ & $0 \mathrm{~mm}$ & $0 \mathrm{~mm}$ & $0 \mathrm{~mm}$ \\
\hline & 0,1 & $0 \mathrm{~mm}$ & $0 \mathrm{~mm}$ & $0 \mathrm{~mm}$ & $0 \mathrm{~mm}$ & $0 \mathrm{~mm}$ & $0 \mathrm{~mm}$ & $0 \mathrm{~mm}$ & $0 \mathrm{~mm}$ & $0 \mathrm{~mm}$ & $0 \mathrm{~mm}$ & $0 \mathrm{~mm}$ & $0 \mathrm{~mm}$ \\
\hline \multirow[t]{4}{*}{ Mecain $^{(}$} & 1,0 & $0 \mathrm{~mm}$ & $0 \mathrm{~mm}$ & $0 \mathrm{~mm}$ & $0 \mathrm{~mm}$ & $0 \mathrm{~mm}$ & $0 \mathrm{~mm}$ & $0 \mathrm{~mm}$ & $0 \mathrm{~mm}$ & $0 \mathrm{~mm}$ & $0 \mathrm{~mm}$ & $0 \mathrm{~mm}$ & $0 \mathrm{~mm}$ \\
\hline & 0,5 & $0 \mathrm{~mm}$ & $0 \mathrm{~mm}$ & $0 \mathrm{~mm}$ & $0 \mathrm{~mm}$ & $0 \mathrm{~mm}$ & $0 \mathrm{~mm}$ & $0 \mathrm{~mm}$ & $0 \mathrm{~mm}$ & $0 \mathrm{~mm}$ & $0 \mathrm{~mm}$ & $0 \mathrm{~mm}$ & $0 \mathrm{~mm}$ \\
\hline & 0,25 & $0 \mathrm{~mm}$ & $0 \mathrm{~mm}$ & $0 \mathrm{~mm}$ & $0 \mathrm{~mm}$ & $0 \mathrm{~mm}$ & $0 \mathrm{~mm}$ & $0 \mathrm{~mm}$ & $0 \mathrm{~mm}$ & $0 \mathrm{~mm}$ & $0 \mathrm{~mm}$ & $0 \mathrm{~mm}$ & $0 \mathrm{~mm}$ \\
\hline & 0,1 & $0 \mathrm{~mm}$ & $0 \mathrm{~mm}$ & $0 \mathrm{~mm}$ & $0 \mathrm{~mm}$ & $0 \mathrm{~mm}$ & $0 \mathrm{~mm}$ & $0 \mathrm{~mm}$ & $0 \mathrm{~mm}$ & $0 \mathrm{~mm}$ & $0 \mathrm{~mm}$ & $0 \mathrm{~mm}$ & $0 \mathrm{~mm}$ \\
\hline \multirow[t]{4}{*}{ Naropin $^{\circledR}$} & 1,0 & $0 \mathrm{~mm}$ & $0 \mathrm{~mm}$ & $0 \mathrm{~mm}$ & $0 \mathrm{~mm}$ & $0 \mathrm{~mm}$ & $0 \mathrm{~mm}$ & $0 \mathrm{~mm}$ & $0 \mathrm{~mm}$ & $0 \mathrm{~mm}$ & $0 \mathrm{~mm}$ & $0 \mathrm{~mm}$ & $0 \mathrm{~mm}$ \\
\hline & 0,5 & $0 \mathrm{~mm}$ & $0 \mathrm{~mm}$ & $0 \mathrm{~mm}$ & $0 \mathrm{~mm}$ & $0 \mathrm{~mm}$ & $0 \mathrm{~mm}$ & $0 \mathrm{~mm}$ & $0 \mathrm{~mm}$ & $0 \mathrm{~mm}$ & $0 \mathrm{~mm}$ & $0 \mathrm{~mm}$ & $0 \mathrm{~mm}$ \\
\hline & 0,25 & $0 \mathrm{~mm}$ & $0 \mathrm{~mm}$ & $0 \mathrm{~mm}$ & $0 \mathrm{~mm}$ & $0 \mathrm{~mm}$ & $0 \mathrm{~mm}$ & $0 \mathrm{~mm}$ & $0 \mathrm{~mm}$ & $0 \mathrm{~mm}$ & $0 \mathrm{~mm}$ & $0 \mathrm{~mm}$ & $0 \mathrm{~mm}$ \\
\hline & 0,1 & $0 \mathrm{~mm}$ & $0 \mathrm{~mm}$ & $0 \mathrm{~mm}$ & $0 \mathrm{~mm}$ & $0 \mathrm{~mm}$ & $0 \mathrm{~mm}$ & $0 \mathrm{~mm}$ & $0 \mathrm{~mm}$ & $0 \mathrm{~mm}$ & $0 \mathrm{~mm}$ & $0 \mathrm{~mm}$ & $0 \mathrm{~mm}$ \\
\hline \multirow[t]{4}{*}{ Ropinaest $^{(\circledR)}$} & 1,0 & $0 \mathrm{~mm}$ & $0 \mathrm{~mm}$ & $0 \mathrm{~mm}$ & $0 \mathrm{~mm}$ & $0 \mathrm{~mm}$ & $0 \mathrm{~mm}$ & $0 \mathrm{~mm}$ & $0 \mathrm{~mm}$ & $0 \mathrm{~mm}$ & $0 \mathrm{~mm}$ & $0 \mathrm{~mm}$ & $0 \mathrm{~mm}$ \\
\hline & 0,5 & $0 \mathrm{~mm}$ & $0 \mathrm{~mm}$ & $0 \mathrm{~mm}$ & $0 \mathrm{~mm}$ & $0 \mathrm{~mm}$ & $0 \mathrm{~mm}$ & $0 \mathrm{~mm}$ & $0 \mathrm{~mm}$ & $0 \mathrm{~mm}$ & $0 \mathrm{~mm}$ & $0 \mathrm{~mm}$ & $0 \mathrm{~mm}$ \\
\hline & 0,25 & $0 \mathrm{~mm}$ & $0 \mathrm{~mm}$ & $0 \mathrm{~mm}$ & $0 \mathrm{~mm}$ & $0 \mathrm{~mm}$ & $0 \mathrm{~mm}$ & $0 \mathrm{~mm}$ & $0 \mathrm{~mm}$ & $0 \mathrm{~mm}$ & $0 \mathrm{~mm}$ & $0 \mathrm{~mm}$ & $0 \mathrm{~mm}$ \\
\hline & 0,1 & $0 \mathrm{~mm}$ & $0 \mathrm{~mm}$ & $0 \mathrm{~mm}$ & $0 \mathrm{~mm}$ & $0 \mathrm{~mm}$ & $0 \mathrm{~mm}$ & $0 \mathrm{~mm}$ & $0 \mathrm{~mm}$ & $0 \mathrm{~mm}$ & $0 \mathrm{~mm}$ & $0 \mathrm{~mm}$ & $0 \mathrm{~mm}$ \\
\hline
\end{tabular}




\section{DISCUSSION}

In clinical practise local anaesthetics are used daily to perform pain free diagnostic procedures. Considering the literature [1], antimicrobial activity of local anaesthetics could lead to false-negative results in microbial assessment of biopsies. Potential mechanisms of these antimicrobial effects are lysis, increased cell wall permeability and dehydrogenase activity [2]. Various studies conclude that prior to diagnostic biopsies the lowest concentration of local agents should be used or a ring block with additive-free local anaesthetics should be performed [1,3]. In 2010, Kose and colleagues [4] showed that under in vivo conditions different local anaesthetics did not have any antimicrobial effects. Also Aydin et al. [5] found no antimicrobial properties of ropivacaine but antimicrobial effectiveness of lidocaine and prilocain was observed. The antibacterial activity of lidocaine was negative in a study performed by Berg et al. [3] but they showed that $\mathrm{EMLA}^{\circledR}$ has a powerful antibacterial effect. Due to these inconsistent results this present study was conducted to evaluate the antimicrobial effects of different commonly used anesthetic agents on Staphylococcus epidermidis, Staphylococcus aureus and Bacillus subtilis. Neither lidocaine, bupivacaine, mepivacaine nor ropivacaine showed an antibacterial effect. We feel that our large sample size allows us to make a valid conclusion.

\section{CONCLUSION}

In summary, neither lidocaine, bupivacaine, mepivacaine nor ropivacaine showed an antibacterial effect on Staphylococcus epidermidis, Staphylococcus aureus and Bacillus subtilis. Due to these findings this local anaesthetics can be used in daily clinical routine to perform pain free diagnostic procedures in which culture specimens are to be obtained. Due to inconsistant results in prior studies we recommend to use the lowest concentration possible of the local anaesthetic, also to avoid other possible side effects of local agents.

\section{ETHICS APPROVAL AND CONSENT TO PARTICIPATE}

Not applicable.

\section{HUMAN AND ANIMAL RIGHTS}

No Animals/Humans were used for studies that are base of this research.

\section{CONFLICT OF INTEREST}

The authors confirm that this article content has no conflict of interest.

\section{ACKNOWLEDGEMENTS}

Declared none.

\section{REFERENCES}

[1] Johnson SM, Saint John BE, Dine AP. Local anesthetics as antimicrobial agents: a review. Surg Infect (Larchmt) $2008 ; 9(2)$ : $205-13$. [http://dx.doi.org/10.1089/sur.2007.036] [PMID: 18426354]

[2] Leung YW, Rawal BD. Mechanism of action of tetracaine hydrochloride against Pseudomonas aeruginosa. J Infect Dis 1977; 136(5): 679-83. [http://dx.doi.org/10.1093/infdis/136.5.679] [PMID: 410897]

[3] Berg JO, Mössner BK, Skov MN, Lauridsen J, Gottrup F, Kolmos HJ. Antibacterial properties of EMLA and lidocaine in wound tissue biopsies for culturing. Wound Repair Regen 2006; 14(5): 581-5. [http://dx.doi.org/10.1111/j.1743-6109.2006.00157.x] [PMID: 17014670]

[4] Kose AA, Karabaggli Y, Kiremitci A, Kocman E, Cetin C. Do local anesthetics have antibacterial effect on Staphylococcus aureus under in vivo conditions? An experimental study. Dermatol Surg 2010; 36(6): 848-52. [http://dx.doi.org/10.1111/j.1524-4725.2010.01559.x] [PMID: 20618369]

[5] Aydin ON, Eyigor M, Aydin N. Antimicrobial activity of ropivacaine and other local anaesthetics. Eur J Anaesthesiol 2001; $18(10)$ : 687-94. [http://dx.doi.org/10.1097/00003643-200110000-00008] [PMID: 11553246]

This is an open access article distributed under the terms of the Creative Commons Attribution 4.0 International Public License (CC-BY 4.0), a copy of which is available at: https://creativecommons.org/licenses/by/4.0/legalcode. This license permits unrestricted use, distribution, and reproduction in any medium, provided the original author and source are credited. 